\title{
Comunicação
}

\section{Seleção de ameixeiras promissoras para a Serra da Mantiqueira}

\author{
Marcelo Caetano de Oliveira ${ }^{1 *}$, Rafael Pio ${ }^{2}$, José Darlan Ramos ${ }^{2}$, Ângelo Albérico Alvarenga ${ }^{3}$ \\ Verônica Andrade dos Santos ${ }^{1}$, Camila Fante ${ }^{4}$
}

\section{RESUMO}

A ameixeira é espécie frutífera, cultivada comercialmente em regiões de inverno ameno a rigoroso, o que torna necessária a seleção de cultivares adequados quanto à adaptação climática. Este trabalho foi conduzido com o objetivo de selecionar materiais genéticos superiores, adaptados às condições edafoclimáticas da região sul de Minas Gerais, nas terras altas da Serra da Mantiqueira. As avaliações foram realizadas em cultivares de ameixeiras da espécie japonesa (Prunus salicina Lindl), 'Pretinha', 'Irati', 'Gema de Ouro', 'Reubennel', 'Roxa de Delfim Moreira', 'Gulfblaze', 'Frontier', 'Santa Rosa', e nove seleções (seleção I, seleção II, seleção III, seleção IV, seleção V, seleção VI, seleção VII, seleção VIII e seleção IX), enxertadas sobre o porta-enxerto 'Okinawa'. O delineamento experimental utilizado foi em blocos casualizados, com oito cultivares e nove seleções de ameixeira, com quatro repetições. Avaliou-se o desenvolvimento vegetativo das plantas, aos 18 e 24 meses após o plantio, no ciclo produtivo 2007/2008, além dos dados fenológico de brotação, florescimento, frutificação e colheita. Conclui-se que as seleções III e IX apresentaram-se como precoces, as seleções VII, VIII, 'Gema de Ouro' e 'Reubennel' apresentaram-se como de meia-estação e os cultivares 'Irati' e 'Gulfblaze' como tardios. Os cultivares Irati, Gema de Ouro e Reubennel demonstraram adaptação às condições edafoclimáticas da região das terras altas da Serra da Mantiqueira de Minas Gerais.

Palavras-chave: Prunus salicina Lindl, ameixa, adaptação climática.

\section{ABSTRACT}

\section{Selection of promising plum cultivars for the Mantiqueira Mountain Range}

Plum (Prunus salicina Lindl) is a fruit species commercially cultivated in regions of mild and severe winters, and cultivar selection is mainly based on climate adaptation. The objective of this study was to select superior genetic materials, adapted to edafoclimatic conditions to the south region of Minas Gerais State, in the highlands of Serra da Mantiqueira. The evaluations were carried out with the plum cultivars 'Pretinha', 'Irati', 'Gema de Ouro', 'Reubennel', 'Roxa de Delfim Moreira', 'Gulfblaze', 'Frontier', 'Santa Rosa', and nine selections (selection I, selection II, selection III, selection IV, selection V, selection VI, selection VII, selection VIII and selection IX), grafted onto the rootstock 'Okinawa'. The experiment was arranged in arandomized block design, with eigth cultivars and nine selections, with four repetitions. Plant vegetative development of eighteen, and twenty-four month old plum trees was evaluated during the crop cycle 2007/2008, besides phenological data on sprouting, blooming, fruiting and harvest. The results

Recebido para publicação em 10/06/2010 e aprovado em 14/07/2011

${ }^{1}$ Engenheiro- Agrônomo, M.Sc., Doutorando em Fitotecnia, Universidade Federal de Lavras (UFLA), Depto. de Agricultura, Caixa Postal 3037, $37200-000$ Lavras, MG, Brasil. caetanocaldas@hotmail.com veronicaandrad@yahoo.com.br

${ }^{2}$ Engenheiro -Agrônomo, D.Sc., Professor Adjunto da Universidade Federal de Lavras (UFLA), Depto. de Agricultura, Caixa Postal 3037, 37200-000 Lavras, MG, Brasil.. Bolsista Produtividade em Pesquisa CNPq. rafaelpio@dag.ufla.br darlan@dag.ufla.br

${ }^{3}$ Engenheiro-Agrônomo, D.Sc., Pesquisador Científico da Empresa de Pesquisa Agropecuária de Minas Gerais (EPAMIG), FELA/UFLA, Caixa Postal 3037, 37200-000 Lavras, MG. angelo@epamig.br

${ }^{4}$ Farmacêutica, M.Sc., Doutoranda em Ciências dos Alimentos, Universidade Federal de Lavras (UFLA), Depto. de Ciências dos Alimentos, Caixa Postal 3037, $37200-000$ Lavras - MG, Brasil. camilafante@yahoo.com.br

*Autor para correspondência. 
showed that the selections III and IX were earlier; selections VII, VIII, 'Gema de Ouro' and 'Reubennel' were midseason; and 'Irati' and 'Gulfblaze' were late. Cultivars Irati, Gema de Ouro and Reubennel showed better adaptation to soil and climatic conditions to the region of the highlands of the Mantiqueira Mountain Range in Minas Gerais.

Key words: Prunus Salicina Lindl, plum, climate adaptation.

\section{INTRODUÇÃO}

As ameixeiras (Prunus sp.) são frutíferas que não suportam calor excessivo, desenvolvendo-se satisfatoriamente na região sul e sudeste do Brasil (Ramos et al., 2003). A necessidade de frio hibernal da ameixeira japonesa (Prunus salicina) varia entre 200 e 1.200 horas abaixo de $7,2^{\circ} \mathrm{C}$, dependendo do cultivar (Herter et al., 1997).

Minas Gerais, em anos passados, já foi considerado o maior produtor de ameixas da região sudeste, mas por causa da incidência da doença "escaldadura das folhas", ocasionada pelo agente Xilella fastidiosa, ocorreu decréscimo significativo nos plantios mineiros. Atualmente, apenas os cultivares Carmesin, Gema de Ouro e Reubennel encontram-se em cultivos comerciais no Estado (Alvarenga et al., 2007).

Visando a selecionar cultivares de ameixeira japonesa para as condições climáticas dessa região, iniciou-se, a partir de agosto de 1994, longo e detalhado levantamento geográfico de áreas onde eventualmente poderiam ser encontradas plantas promissoras de ameixeira. Foi detectada área em propriedade particular, no município de Wenceslau Braz, situado na serra da Mantiqueira do sul do Estado, em altitude de $1.005 \mathrm{~m}$. Nesse local, foram encontradas plantas remanescentes de antigo pomar comercial, oriundo de indivíduos provenientes da propagação sexuada e assexuada. Porém, as plantas estavam produzindo sob condições adversas, sem quaisquer tratos culturais. Após período de avaliação de dois ciclos produtivos (1996-1997 e 1997-1998), foram selecionadas plantas com características favoráveis, principalmente com relação às épocas de floração, maturação dos frutos e sua qualidade comercial. Essas seleções promissoras foram catalogadas e enxertadas sobre o pessegueiro 'Okinawa' e alocadas na Fazenda Experimental de Maria da Fé, da Empresa de Pesquisa Agropecuária de Minas Gerais (FEMF/EPAMIG) (Ramos et al., 2007).

Em condições adequadas, a ameixeira japonesa floresce abundantemente, com a densidade de flores, podendo chegar ao redor de 40 flores para cada $25 \mathrm{~cm}$ linear de ramo. Acredita-se que o vingamento de 5\% das flores fecundadas seja suficiente para assegurar boa produção comercial (Carvalho \& Raseira, 1990).
O período de florescimento varia, nos cultivares de ameixeira japonesa, em função da região de cultivo e das características próprias de cada cultivar. Nos cultivares Grancuore, Kelsey 31, Gema de Ouro e Januária, foi observada floração entre agosto e setembro, em São Paulo (Barbosa et al., 1991). No Rio Grande do Sul, Grellmann \& Simonetto (1995) observaram o florescimento nos cultivares Reubennel, Harry Pickstone e Santa Rosa nos meses de julho a setembro.

O objetivo deste trabalho foi selecionar ameixeiras superiores para as condições edafoclimáticas das terras altas da Serra da Mantiqueira, sul do Estado de Minas Gerais.

\section{MATERIAL E MÉTODOS}

A pesquisa foi realizada na Fazenda Experimental de Maria da Fé (FEMF/EPAMIG), Maria da Fé-MG, microrregião da Serra da Mantiqueira no sul do Estado de Minas Gerais, no período de outubro de 2007 a dezembro de 2008. O local situa-se a $22^{\circ} 18^{\prime}$ de latitude Sul e $45^{\circ} 23^{\prime}$ de longitude Oeste, altitude média de $1.276 \mathrm{~m}$, clima Mesotérmico, com temperatura média de $17^{\circ} \mathrm{C}$ e precipitação em torno de $1.738,6 \mathrm{~mm}$ anuais.

Mudas dos cultivares Pretinha, Irati, Gema de Ouro, Reubennel, Roxa de Delfim Moreira, Gulfblaze, Frontier, Santa Rita e nove seleções promissoras (seleção I, seleção II, seleção III, seleção IV, seleção V, seleção VI, seleção VII, seleção VIII e seleção IX), enxertadas sobre o porta-enxerto 'Okinawa', foram plantadas a campo, no espaçamento 6 x $4 \mathrm{~m}$, em sistema de condução tipo vaso com quatro pernadas. A área de plantio passou por calagem, visando à correção do solo; em seguida, as covas foram preparadas com 60 dias de antecedência ao plantio, com $0,5 \mathrm{~kg}$ de calcário dolomítico, $800 \mathrm{~g}$ de superfosfato simples, 300 gramas de cloreto de potássio, 20 litros de esterco de gado curtido; posteriormente, foram realizadas três coberturas com $100 \mathrm{~g}$ por cova da formulação $20-00-20$, a cada 30 dias, até ao final do período das chuvas.

O delineamento utilizado foi em blocos ao acaso, com 17 tratamentos, quatro blocos e seis plantas por parcela, sendo avaliadas as quatro centrais. Durante a condução, as plantas foram criteriosamente adubadas, segundo análise do solo e pulverizadas sistematicamente com defensivos agrícolas 
específicos, conforme recomendação de Alvarenga et al. (2007). Durante o inverno dos ciclos 2007/2008, as plantas foram podadas e pulverizadas com calda bordalesa.

Foi realizada a caracterização do desenvolvimento vegetativo das plantas, a partir de sua altura (do solo até extremidade apical), diâmetro abaixo e acima do ponto de enxertia (cinco cm acima e abaixo), avaliados aos 18 e 24 meses após o plantio. Na safra de 2007/2008, foram coletados os seguintes dados fenológicos: emissão de brotação, início da floração (5\% de flores abertas), final da floração (mais de $90 \%$ de flores abertas), início da frutificação e data da colheita. Para isso, foram realizadas observações diárias nos meses de julho a dezembro do ciclo de 2007/2008.

Os dados referentes à caracterização vegetativa foram submetidos à análise de variância e comparados pelo teste de médias Scott-Knott ( $\mathrm{p}<0,05)$. As análises foram realizadas pelo programa computacional Sistema para Análise de Variância - SISVAR.

\section{RESULTADOS E DISCUSSÃO}

Nas avaliações realizadas aos 18 e 24 meses, constatou-se que os cultivares Irati, Gema de Ouro, Reubennel, Gulfblaze, Santa Rosa e as seleções V e IX agruparam-se entre aquelas de maior altura em ambas as avaliações (Tabela 1). Entretanto, ressalta-se também que, no grupo de maior desenvolvimento em altura, para os 24 meses após o plantio ,estavam os genótipos Frontier, Seleção II, Seleção III, Seleção IV.

Esses resultados são importantes no sentido de indicar genótipos adaptados às condições edafoclimáticas da região em questão, demonstrando adaptabilidade e vigor vegetativo, o que favorece formação precoce do pomar, ampliando, com isso, a diversidade varietal aos produtores locais.

Para o diâmetro abaixo do ponto de enxertia, na avaliação realizada aos 18 meses, destacaram-se os cultivares Gema de Ouro, Reubennel, Gulfblaze, Santa Rosa, Seleção VIII e Seleção IX, enquanto, aos 24 meses, somente o cultivar Irati apresentou maior diâmetro (Tabela 1). Já para o diâmetro acima do ponto de enxertia, nas duas avaliações, os cultivares Irati, Reubennel, Gulfblaze, seleção III e IX apresentaram maior diâmetro em ambas as avaliações (Tabela 1). Com relação às diferenças observadas nos diâmetros abaixo e acima do ponto de enxertia, de maneira geral, o desenvolvimento apresentado não caracterizou problema relacionado com a incompatibilidade entre os genótipos e o porta-enxerto 'Okinawa'.

Quanto à descrição fenológica dos cultivares e seleções, constatou-se que os cultivares Gulfblaze e Frontier e as seleções II, V e VIII iniciaram a emissão das brotações antes do florescimento (Tabela 1). Isso é um indicativo de baixa adaptação às condições climáticas do local, uma vez que as ameixeiras japonesas florescem antes ou junto com a emissão das brotações, já que as gemas floríferas

Tabela 1. Média da altura de planta (AP, $\mathrm{cm})$, diâmetro abaixo do ponto de enxertia $(\otimes \mathrm{AB}, \mathrm{mm})$ e diâmetro acima do ponto de enxertia $(\otimes \mathrm{AC}, \mathrm{mm})$ em cultivares e seleções promissoras de ameixeira japonesa (Prunus salicina), enxertadas sobre o porta-enxerto 'Okinawa', 18 e 24 meses após a implantação

\begin{tabular}{|c|c|c|c|c|c|c|}
\hline \multirow{2}{*}{$\begin{array}{l}\text { Cultivares e seleções } \\
\text { de ameixeira }\end{array}$} & \multicolumn{3}{|c|}{18 meses pós- plantio } & \multicolumn{3}{|c|}{24 meses pós- plantio } \\
\hline & $\mathbf{A P}(\mathbf{c m})$ & $\begin{array}{r}\otimes \mathrm{AB} \\
(\mathrm{mm})\end{array}$ & $\begin{array}{r}\otimes \mathrm{AC} \\
(\mathbf{m m})\end{array}$ & $\mathbf{A P}(\mathbf{c m})$ & $\begin{array}{l}\otimes \mathrm{AB} \\
(\mathrm{mm})\end{array}$ & $\begin{array}{l}\otimes \mathrm{AC} \\
(\mathrm{mm})\end{array}$ \\
\hline Pretinha & $67,8 \mathrm{~b}$ & $11,3 \mathrm{~b}$ & $9,0 \mathrm{~b}$ & $90,0 \mathrm{c}$ & $15,5 \mathrm{~d}$ & $26,0 \mathrm{~b}$ \\
\hline Irati & $137,2 \mathrm{a}$ & $21,5 \mathrm{a}$ & $18,6 \mathrm{a}$ & $180,0 \mathrm{a}$ & $53,6 \mathrm{a}$ & $125,0 \mathrm{a}$ \\
\hline Gema de Ouro & $127,7 \mathrm{a}$ & $17,3 \mathrm{a}$ & $15,0 \mathrm{a}$ & $187,5 \mathrm{a}$ & $35,9 \mathrm{~b}$ & $75,0 \mathrm{~b}$ \\
\hline Reubennel & $118,2 \mathrm{a}$ & $17,4 \mathrm{a}$ & $16,6 \mathrm{a}$ & $195,0 \mathrm{a}$ & $41,1 \mathrm{~b}$ & $96,2 \mathrm{a}$ \\
\hline Roxa de Delfim Moreira & $91,0 \mathrm{~b}$ & $12,0 \mathrm{~b}$ & $10,3 \mathrm{~b}$ & $145,0 \mathrm{~b}$ & $33,6 \mathrm{~b}$ & $80,0 \mathrm{~b}$ \\
\hline Gulfblaze & $115,7 \mathrm{a}$ & $18,9 \mathrm{a}$ & $16,4 \mathrm{a}$ & $145,7 \mathrm{a}$ & $23,2 \mathrm{c}$ & $104,7 \mathrm{a}$ \\
\hline Frontier & $59,7 \mathrm{~b}$ & $9,5 \mathrm{~b}$ & $16,4 \mathrm{a}$ & $152,5 \mathrm{a}$ & $23,4 \mathrm{c}$ & $72,5 \mathrm{~b}$ \\
\hline Santa Rosa & $123,0 \mathrm{a}$ & $16,0 \mathrm{a}$ & $14,5 \mathrm{a}$ & $170,0 \mathrm{a}$ & $33,7 \mathrm{~b}$ & $70,0 \mathrm{~b}$ \\
\hline Seleção I & $69,5 \mathrm{~b}$ & $10,8 \mathrm{~b}$ & $9,8 \mathrm{~b}$ & $109,0 \mathrm{c}$ & $25,8 \mathrm{c}$ & $44,3 \mathrm{c}$ \\
\hline Seleção II & $85,2 \mathrm{~b}$ & $13,5 \mathrm{~b}$ & $11,5 \mathrm{~b}$ & $145,0 \mathrm{a}$ & $30,3 \mathrm{~b}$ & $48,7 \mathrm{c}$ \\
\hline Seleção III & $65,7 \mathrm{~b}$ & $11,0 \mathrm{~b}$ & $8,2 \mathrm{~b}$ & $150,0 \mathrm{a}$ & $31,3 \mathrm{~b}$ & $103,7 \mathrm{a}$ \\
\hline Seleção IV & $95,7 \mathrm{~b}$ & $15,4 \mathrm{~b}$ & $12,0 \mathrm{~b}$ & $162,0 \mathrm{a}$ & $33,5 \mathrm{~b}$ & $71,2 b$ \\
\hline Seleção V & $87,2 \mathrm{a}$ & $12,0 \mathrm{~b}$ & $10,8 \mathrm{~b}$ & $180,0 \mathrm{a}$ & $31,9 \mathrm{~b}$ & $70,0 \mathrm{~b}$ \\
\hline Seleção VI & $63,5 \mathrm{~b}$ & $8,7 \mathrm{~b}$ & $7,8 \mathrm{~b}$ & $113,3 \mathrm{c}$ & $15,6 \mathrm{~d}$ & $48,7 \mathrm{c}$ \\
\hline Seleção VII & $70,5 \mathrm{~b}$ & $11,8 \mathrm{~b}$ & $9,6 \mathrm{~b}$ & $132,5 \mathrm{~b}$ & $27,1 \mathrm{c}$ & $52,5 \mathrm{c}$ \\
\hline Seleção VIII & $121,7 \mathrm{a}$ & $15,9 \mathrm{a}$ & $13,6 \mathrm{a}$ & $136,2 \mathrm{~b}$ & $31,5 \mathrm{~b}$ & $81,2 \mathrm{~b}$ \\
\hline Seleção IX & $97,5 \mathrm{a}$ & $14,9 \mathrm{a}$ & $11,3 \mathrm{~b}$ & $151,2 \mathrm{a}$ & $37,1 \mathrm{~b}$ & $135,0 \mathrm{a}$ \\
\hline $\mathrm{CV}(\%)$ & 30,1 & 21,9 & 26,5 & 18,1 & 22,3 & 33,4 \\
\hline
\end{tabular}

Médias seguidas de mesmas letras na coluna, para cada variável, não diferem entre si pelo teste de Scott-Knott, a 5\% de probabilidade de erro. 
possuem menor necessidade em frio hibernal para florescer (Alvarenga et al., 2007).

O resultado, observado para o cultivar Gema de Ouro, está de acordo com o constatado por Barbosa et al. (1991) que observaram a floração para este cultivar em agosto/ setembro, no Estado de São Paulo. Nas condições do Rio Grande do Sul, o florescimento nos cultivares Reubennel e Santa Rosa, ocorre nos meses de julho/agosto e agosto/setembro, respectivamente (Grellmann \& Simonetto, 1995).

Resultados, obtidos por Silva (2000), demonstraram que os cultivares Reubennel, Gema de Ouro, Roxa de Itaquera, Grancuore e Irati apresentaram floração precoce entre a segunda quinzena de julho e a primeira de agosto, enquanto o cultivar Roxa Japonesa apresentou comportamento tardio, com floração na segunda quinzena de agosto.

Estes resultados são significativos no sentido de oferecer ao produtor materiais com ciclos reprodutivos diferentes, aumentando o período de oferta do produto ao mercado, além de diversificar as opções de produto ao consumidor.

Verificou-se que o cultivar Pretinha e a seleção VI não floresceram, possivelmente por necessitarem de maior acúmulo de frio, não tendo sua necessidade suprida na região. Porém, os cultivares Roxa de Delfim Moreira, Frontier, Santa Rosa e as seleções I, II, IV e V não frutificaram, não sendo recomendado, assim, seu uso para região.

Quanto à época de colheita, as seleções III e IX apresentaram-se como precoces, as seleções VII, VIII, 'Gema de Ouro' e 'Reubennel', como de meia-estação, e os cultivares 'Irati' e 'Gulfblaze', como tardios (Tabela 2). Assim, esses cultivares e as seleções citadas são boas opções, visando à colheita escalonada, no mês de dezembro, nas condições das terras altas da Serra da Mantiqueira de Minas Gerais, mês em que há maior demanda de ameixa, por ser fruta tipicamente natalina, possibilitando, ainda, preços de mercado superiores, para o produtor.

Tabela 2. Emissão das brotações, período de floração (início e término), frutificação e colheita na safra 2008 de cultivares e seleções promissoras de ameixeira japonesa (Prunus salicina), enxertadas sobre o porta-enxerto 'Okinawa'

\begin{tabular}{|c|c|c|c|c|c|}
\hline \multirow{2}{*}{$\begin{array}{l}\text { Cultivares e seleções } \\
\text { de ameixeira }\end{array}$} & \multirow{2}{*}{$\begin{array}{c}\text { Emissão das } \\
\text { brotações }\end{array}$} & \multicolumn{2}{|c|}{ Período de floração } & \multirow{2}{*}{ Frutificação } & \multirow{2}{*}{ Colheita } \\
\hline & & Início & Término & & \\
\hline Pretinha & $20 / 08$ & $*$ & $*$ & $* *$ & $* *$ \\
\hline Irati & $21 / 08$ & $21 / 08$ & $17 / 10$ & $05 / 10$ & $17 / 12$ \\
\hline Gema de Ouro & $26 / 08$ & $26 / 08$ & $24 / 10$ & $24 / 10$ & $10 / 12$ \\
\hline Reubennel & $19 / 09$ & $21 / 08$ & $10 / 10$ & $05 / 09$ & $13 / 12$ \\
\hline Roxa de Delfim Moreira & $19 / 09$ & $16 / 09$ & $24 / 10$ & $* *$ & $* *$ \\
\hline Gulfblaze & 01/08 & $20 / 08$ & $27 / 10$ & $29 / 09$ & $20 / 12$ \\
\hline Frontier & $26 / 08$ & $16 / 09$ & $20 / 10$ & $* *$ & $* *$ \\
\hline Santa Rosa & $16 / 09$ & $16 / 09$ & $30 / 10$ & $* *$ & $* *$ \\
\hline Seleção I & $10 / 09$ & 03/09 & $19 / 10$ & $* *$ & $* *$ \\
\hline Seleção II & $10 / 09$ & $16 / 09$ & $24 / 10$ & $* *$ & $* *$ \\
\hline Seleção III & $10 / 09$ & $21 / 08$ & $15 / 11$ & $30 / 10$ & $05 / 12$ \\
\hline Seleção IV & $10 / 09$ & $10 / 09$ & $24 / 10$ & $* *$ & $* *$ \\
\hline Seleção V & 03/09 & $19 / 09$ & $19 / 10$ & $* *$ & $* *$ \\
\hline Seleção VI & $26 / 08$ & $*$ & $*$ & $* *$ & $* *$ \\
\hline Seleção VII & $10 / 09$ & $10 / 09$ & $16 / 10$ & $23 / 09$ & $10 / 12$ \\
\hline Seleção VIII & $10 / 09$ & $16 / 09$ & $20 / 10$ & $24 / 09$ & $10 / 12$ \\
\hline Seleção IX & $21 / 08$ & $21 / 08$ & $17 / 10$ & $16 / 10$ & $07 / 12$ \\
\hline
\end{tabular}

*Não floresceu; ** Não frutificou.

\section{CONCLUSÕES}

As seleções III e IX apresentaram-se como precoces, as seleções VII, VIII, os cultivares 'Gema de Ouro' e 'Reubennel' apresentaram-se como de meia-estação e os cultivares Irati e Gulfblaze, como tardios.

Os cultivares Irati, Gema de Ouro e Reubennel demonstraram adaptação às condições edafoclimáticas da região das terras altas da Serra da Mantiqueira de Minas Gerais.

\section{REFERÊNCIAS}

Alvarenga AA, Abrahao E, Carvalho VL, Silva RA, Fraguas JC, Cunha RL, Santa Cecilia LVC, Silva VJ. (2007) Pêssego, nectarina e ameixa (Prunus spp.). In: TRAZILBO, J.P.Jr., MADELAINE V. (Org.). 101 Culturas - Manual de tecnologias agrícolas. Belo Horizonte: EPAMIG, p.611-624.

Barbosa W, Dall'orto FAC, Ojima M, Martins FP, Santos RR, Sabino JC (1991) Polinização das fruteiras de caroço: Ameixeira, Nectarineira e Pessegueiro. O Agronômico, 43:3-13.

Carvalho TCP, Raseira MCB (1990) Aspectos relacionados a polinização e autocompatibilidade em ameixeira japonesa (Prunus salicina Lindl.). HortiSul, 2:29-32. 
Grellmann EO, Simonetto PR (1995) Dados de fenologia e produção de cultivares de ameixeira. Circular Técnica. FEPAGRO, $11 \mathrm{p}$.

Herter FG, Zanol GC, Reisser JC (1997) Pessegueiro e Ameixeira. Informe Agropecuário, 18:18-23.

Ramos JD, Hafle OM, Chalfun NNJ, Souza HA, Cavallari LL (2007) Seleção de clones de ameixeira para o sul do estado de Minas Gerais. Revista Brasileira de Fruticultura, 29:559-562.
Ramos JD, Matos LES, Gontijo TCA, Pio R, Junqueira KP Santos, FC (2003) Enraizamento de estacas herbáceas de 'Mirabolano' (Prunus cerasifera Ehrn) em diferentes substratos e concentrações de ácido indolbutírico. Revista Brasileira de Fruticultura, 25:189-191.

Silva FP (2000) Comportamento de cultivares de ameixeira (Prunus salicina Lindl.) em Caldas-MG. Tese Doutorado. Universidade Federal de Lavras, Lavras, 93p. 